\title{
The nature of the light variability of the silicon star HR $7224^{\star}$
}

\author{
J. Krtička ${ }^{1}$, Z. Mikulášek ${ }^{1,2}$, G. W. Henry ${ }^{3}$, J. Zverko ${ }^{4}$, J. Žižňovský ${ }^{4}$, J. Skalický ${ }^{1}$, and P. Zvěřina ${ }^{1}$ \\ 1 Department of Theoretical Physics and Astrophysics, Masaryk University, Kotlářská 2, 61137 Brno, Czech Republic \\ e-mail: krticka@physics.muni.cz \\ 2 Observatory and Planetarium of J. Palisa, VŠB - Technical University, Ostrava, Czech Republic \\ 3 Center of Excellence in Information Systems, Tennessee State University, Nashville, Tennessee, USA \\ 4 Astronomical Institute, Slovak Academy of Sciences, Tatranská Lomnica, Slovak Republic
}

Received 9 October 2008 / Accepted 16 February 2009

ABSTRACT

\begin{abstract}
Context. Although photometric variations of chemically peculiar $(\mathrm{CP})$ stars are frequently used to determine their rotational periods, the detailed mechanism of their light variability remains poorly understood.

Aims. We simulate the light variability of the star HR 7224 using the observed surface distribution of silicon and iron.

Methods. We used the TLUSTY model atmospheres calculated for the appropriate silicon and iron abundances to obtain the emergent flux and to predict the rotationally modulated light curve of the star. We also obtained additional photometric measurements and employed our own regression procedure to derive a more precise estimate of the light elements.

Results. We show that the light variation of the star can be explained as a result of i) the uneven surface distribution of the elements, ii) the flux redistribution from the ultraviolet to the visible part of the spectrum, and iii) rotation of the star. We show that the silicon bound-free transitions and iron bound-bound transitions provide the main contribution to the flux redistribution, although an additional source of opacity is needed. We confirm that numerous iron lines significantly contribute to the well-known depression at $5200 \AA$ and discuss the connection between iron abundance and the value of peculiarity index $a$.

Conclusions. The uneven surface distribution of silicon and iron is able to explain most of the rotationally modulated light variation in the star HR 7224.
\end{abstract}

Key words. stars: chemically peculiar - stars: early type - stars: variables: general - stars: individual: HR 7224

\section{Introduction}

Chemically peculiar (CP) stars represent a large class of upper main sequence stars where the processes of radiative diffusion and gravitational settling in their atmospheres give rise to pronounced deviations in the chemical composition of these stars from the solar value (Vauclair 2003; Michaud 2005). The CP stars are natural laboratories for testing modern model atmospheres thanks to the unusual chemistry with rather strong under- or overabundance of some elements. The application of advanced modelling techniques, such as model atmospheres with magnetic fields (e.g., Kochukhov et al. 2005; Khan \& Shulyak 2006), radiative diffusion codes, and Doppler imaging techniques (Khokhlova et al. 2000), provides us with detailed information about the surface structure of these stars. Despite these fascinating advances in their study during recent decades, the light variability of CP stars is still poorly understood.

Some CP stars show inhomogeneous surface distribution of chemical elements on their surface (e.g., Khokhlova et al. 2000) as determined from rotationally modulated spectral line variability (see, e.g., Lehmann et al. 2006). The uneven surface distribution of various elements, together with rotation, has been presumed to be the origin of these stars' light variability. However, the details of this mechanism have not been determined. Line blanketing by multiple lines of overabundant elements (mainly

\footnotetext{
* New obtained light curves are only available in electronic form at the CDS via anonymous ftp to

cdsarc.u-strasbg.fr $(130.79 .128 .5)$ or via

http://cdsweb.u-strasbg.fr/cgi-bin/qcat?J/A+A/499/567
}

iron) and the flux redistribution induced by these lines has been proposed as one of the causes of the light variability (Molnar 1973). Other mechanisms proposed include the influence of bound-free transitions (Peterson 1970; Lanz et al. 1996), surface temperature differences or variable temperature gradients (Kodaira 1967; Weiss et al. 1976), and the presence of magnetic fields (e.g., Kochukhov et al. 2005). Finally, circumstellar matter, if present, may also influence the light curves (Landstreet \& Borra 1978; Nakajima 1985; Smith \& Groote 2001; Townsend et al. 2005).

One of the first attemps to simulate the light variability of CP stars was done by Krivosheina et al. (1980), who reproduced the light curve of the CP star CU Vir. However, detailed modelling of CP star light variability had to await precise model atmospheres (e.g., Lanz \& Hubeny 2007), improved opacity data (Seaton et al. 1992), and much faster computers. Krtička et al. (2007) took advantage of these tools and used the surface maps of Khokhlova et al. (2000) to simulate the light curve of HD 37776 successfully. They demonstrate that the inhomogeneous surface distribution of silicon and helium, along with the bound-free transitions of these elements, accounted for most of the light variability in this star.

Much work remains to be done to understand the light variability in CP stars. In particular, the role of iron, which is found to be significantly underabundant in the atmosphere of HD 37776 (Khokhlova et al. 2000), remains to be clarified. For this purpose we selected the silicon star HR 7224, whose silicon and iron surface distributions were derived via Doppler imaging by Lehmann et al. (2007). 


\section{CP star HR 7224}

HR 7224 (HD 177410, HIP 93187, EE Dra) is a rapidly rotating CP star classified as A0p Si by Cowley et al. (1969). Molnar (1972) classified HR 7224 as a B9.5 IIIp Si star, based on lowresolution spectroscopy. Lehmann et al. (2006, 2007) found that the star has enhanced $\mathrm{Si}$ and $\mathrm{Fe}$ abundances, whereas $\mathrm{He}$ is extremely depleted; they classified it as a helium-weak silicon CP star.

Winzer (1974) found photometric variability in HR 7224 and determined a period of 1.1663 from $48 U B V$ observations done in 1970-72. Adelman (1997) derived a new period of 1.123095 based on 616 Strömgren $u v b y$ observations taken in the 1993-94 and 1994-95 observing seasons with the Four College Automated Photometric Telescope (FCAPT) along with the older $V$ measurements of Winzer (1974). Hipparcos obtained $409 \mathrm{Hp}, \mathrm{B}_{\mathrm{T}}$, and $V_{\mathrm{T}}$ observations in 1989-92 (ESA 1997), and ESA (1998) determined the photometric period to be 1.123248 .

All of the optical light curves phase together fairly well and show a double-wave with two maxima of different height (see Fig. 1). HR 7224 was recently classified from the shape of its light curve as a prototype of a photometrically simple CP star with a double-wave light curve with two unequally prominent bright spots centred on the phases $\varphi=0.0$ and 0.5 (Mikulášek et al. 2008a). There are only two attempts to measure the magnetic field of HR 7224 available in the literature (Bohlender et al. 1993; Lehmann et al. 2007); both produced negative results. Recent magnetic field measurements (Kudryavtsev, private communication) also gave negative results, however the observations are still continuing to cover the whole rotational cycle.

Adelman (2004) reported an unprecedented change in the photometric behaviour of HR 7224. Comparing time series observations taken with FCAPT before 1996 with those taken in 2003 , he found that the amplitude of variability had increased from a typical value of $0.04 \mathrm{mag}$ to $0.21 \mathrm{mag}$. He also reported that the period of variability had changed from $1^{\mathrm{d}} .123$ to 101 days. Unfortunately, the photometric data from this critical era have not been published.

Adelman's astonishing result, along with the lack of available high-resolution spectroscopy of HR 7224, motivated Lehmann et al. (2006) to carry out an extensive spectroscopic observing program on the star. Their 564 high-resolution spectrograms allow determination of radial velocities via cross correlation to an accuracy better than $100 \mathrm{~m} \mathrm{~s}^{-1}$. They found radial velocity variations in this swiftly rotating star with an amplitude of $15 \mathrm{~km} \mathrm{~s}^{-1}$ and a period of $1.123248(9)$, in excellent agreement with the previous determination by ESA (1998). They found no further periodicities, in particular nothing around the 101-day period of Adelman (2004).

Lehmann et al. (2007) used the same 564 spectrograms to derive Doppler images of surface elemental distributions. Their map of the silicon and iron abundance on the surface of HR 7224 is an ideal starting point for the simulation of the star's expected light curves. To avoid any difficulties phasing the spectroscopic and photometric observations together, we acquired new photometric observations to improve the rotational ephemeris of HR 7224.

\subsection{New BV photometry of HR 7224}

Our new $B V$ photometry was acquired between March and May 2008 with the T3 $0.4 \mathrm{~m}$ automatic photoelectric telescope (APT) at Fairborn Observatory. This APT uses a temperature-stabilised EMI 9924B photomultiplier tube to measure photon count rates

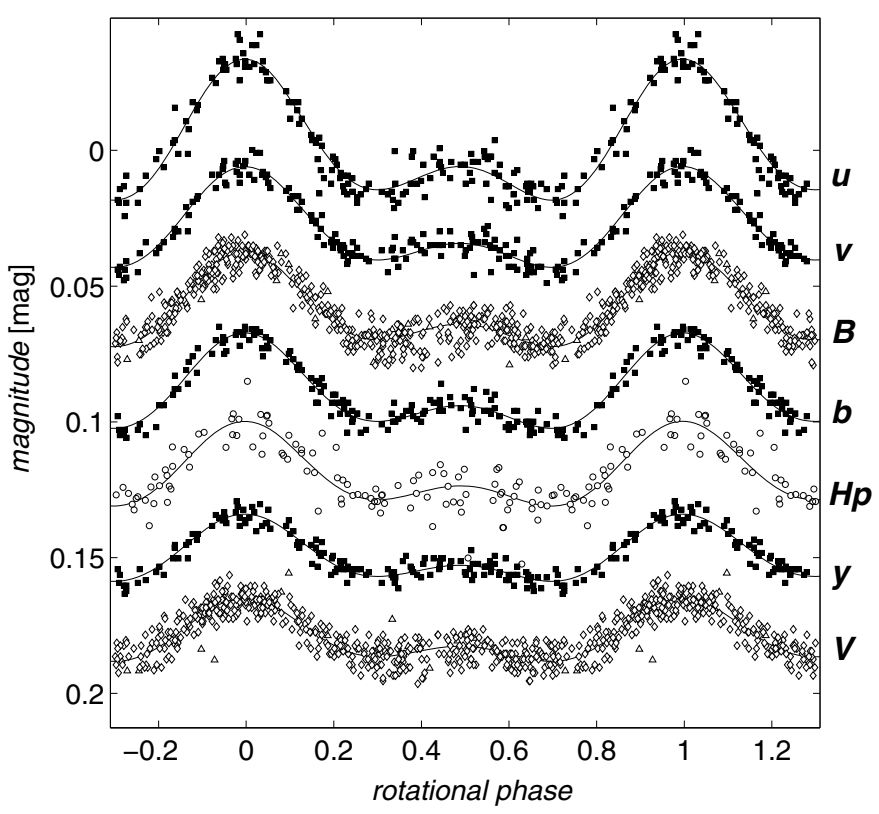

Fig. 1. Photometric observations of HR 7224 plotted as a function of the new linear phase in Eq. (3). Data are from Winzer (1974), $B V(\triangle)$; Adelman (1997), uvby (); Hipparcos (ESA 1997), Hp (o), and the T3 APT (this paper), $B V(\diamond)$. Solid lines denote the fit according to Eq. (1). $B_{\mathrm{T}} V_{\mathrm{T}}$ and $U$ magnitudes are not plotted here due to their large scatter.

through Johnson $B$ and $V$ filters. HR 7224 and its comparison stars were measured in the following sequence, termed a group observation: $\mathrm{K}-\mathrm{S}-\mathrm{C}-\mathrm{V}-\mathrm{C}-\mathrm{V}-\mathrm{C}-\mathrm{V}-\mathrm{C}-\mathrm{S}-\mathrm{K}$, where $\mathrm{K}$ is the check star (HD 172728, $V=5.74, B-V=-0.045, \mathrm{~A} 0 \mathrm{~V}), \mathrm{C}$ is the comparison star (HD 172569, $V=6.07, B-V=0.279, \mathrm{~F} 0 \mathrm{~V}$ ), $\mathrm{V}$ is HR 7224, and S is a sky measurement. Three V-C and two K-C differential magnitudes are formed from each sequence and averaged together to create group mean differential magnitudes. The typical precision of a group mean is $0.003-0.004$ mag for this telescope. To filter out observations taken in non-photometric conditions, group means with a standard deviation greater than $0.01 \mathrm{mag}$ were discarded. From the 572 group observations collected by the telescope, $478 \mathrm{~B}$ and $462 \mathrm{~V}$ group means survived the filtering process and were used in this analysis.

The surviving group means were corrected for differential extinction with nightly extinction coefficients, transformed to the Johnson system with yearly-mean transformation coefficients, and treated as single observations thereafter. Up to five group observations were acquired each clear night at intervals of approximately two hours. Further information on the operation of the APT and the analysis of the data can be found in Henry (1995a,b) and Eaton et al. (2003).

The photometric data used in this analysis are available through SIMBAD and the On-line database of photometric observations of $m C P$ stars (http: //astro.physics .muni.cz/ mcpod).

\subsection{New linear ephemeris}

To derive the ephemeris, we used all available photometric data on HR 7224 including the $U B V$ measurements of Winzer (1974), the $H p$ and $B_{\mathrm{T}} V_{\mathrm{T}}$ observations from Hipparcos (ESA 1997), the Strömgren uvby photometry by Adelman (1997), and the T3 APT $B V$ observations in this paper. The total of 2013 individual photometric measurements covers a time span of 
38 years; they were supplemented with the 564 measurements of silicon line equivalent widths $(E W)$ obtained by Lehmann et al. (2007) in 2004-05 and kindly provided to us by Dr. H. Lehmann.

The analysis of these data to determine the ephemeris is described in Mikulášek et al. (2008b). It is assumed that all the photometric and spectroscopic variability can be expressed in terms a simple regression model.

\subsubsection{Regression model and robust regression procedure}

The formulation of the observed phase variation of the light and equivalent widths of silicon lines in terms of a general regression model is relatively simple since the light and $E W$ phase curves are similar in shape, though shifts may exist in some cases. Consequently, we can express them with the following relations:

$y_{c j}\left(t_{i}\right)=\bar{y}_{c j}+\frac{1}{2} A_{c} F\left(\vartheta_{i j}\right), \quad \vartheta_{i j}=\left(t_{i}-M_{0}-\Delta M_{0 j}\right) / P$,

where $y_{c j}$ is the predicted value of an observed quantity (magnitude, $E W$ ) in a colour (pass band) $c$ (in this case $u, U, v, B, b, H p, y, V$, and $E W$ ) of the jth set of measurements (Winzer, Hipparcos, Adelman I and II, Lehmann, and this paper) at the particular $\mathrm{JD}_{\text {hel }}$ instant $t_{i}$, and $\bar{y}_{c j}$ is the weightedmean value of the quantity. Note that observations in $B_{\mathrm{T}}$ were included into $B$ and $V_{\mathrm{T}}$ into $V$. Here $A_{c}$ is an "effective amplitude", the parameter robustly describing the measure of the variability in the colour $c$, and $F(\vartheta)$ is a normalised (unique) function expressing the form of the observed phase curves (for details see Mikulášek et al. 2007a,b). It is a periodic function of the monotonically growing "phase function" $\vartheta$ introduced by Mikulášek et al. (2008b).

The phase function of time $\vartheta(t)$ is determined by the ephemeris, which is given in the second part of Eq. (1). We assume that the period $P$ of HR 7224 is constant and define zero phase to be the light maximum $M_{0}$ that is situated nearest to the weighted centre of gravity of measurements used for the ephemeris determination. $\Delta M_{0 j}$ are free parameters correcting $M_{0}$ for some groups of observation. We applied them only when justified, particularly for observations of Winzer (1974; see the following discussion) and for the equivalent widths of the Si lines.

Function $F(\vartheta)$ is the simplest normalised periodic function that represents the observed photometric and spectroscopic variations of HR 7224 in detail. The phase of maximum brightness is defined to be 0.0 , and the amplitude is defined to be 1.0 . The function, being the sum of three orthogonal terms, is described by two parameters $a_{1}$ and $a_{2}$. The first parameter quantifies the symmetric portion of the deviation of the light curve from a simple cosine course; the second parameter expresses any asymmetry in the light curve:

$$
\begin{aligned}
F\left(\vartheta, a_{1}, a_{2}\right)= & \sqrt{1-a_{1}^{2}-a_{2}^{2}} \cos (2 \pi \vartheta)+a_{1} \cos (4 \pi \vartheta) \\
& +a_{2}\left[\frac{2}{\sqrt{5}} \sin (2 \pi \vartheta)-\frac{1}{\sqrt{5}} \sin (4 \pi \vartheta)\right]
\end{aligned}
$$

\subsubsection{New findings}

We used the same robust, iterative, regression procedure described in Sect. 3.3 of Mikulášek et al. (2008b) to determine a new linear ephemeris for HR 7224. The ephemeris consists of the period $P$, the $\mathrm{JD}_{\text {hel }}$ of the light curve maximum $M_{0}$, the mean shift of Winzer's measurements $\Delta M_{0 \text { win }}$, and the mean shift of

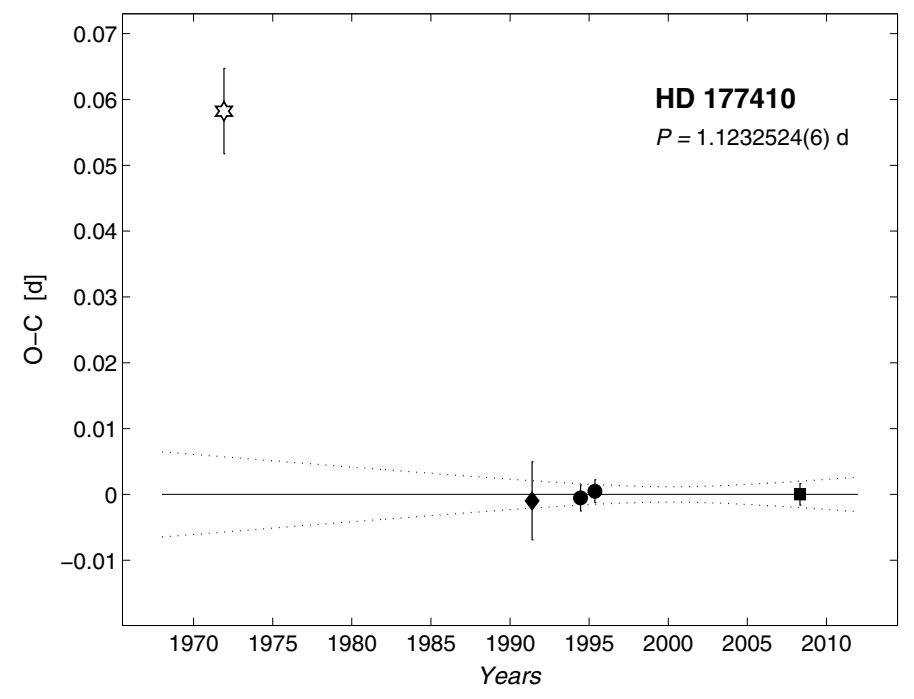

Fig. 2. O-C diagram of HR 7224 computed with the new linear ephemeris in Eq. (3). The open star represents the observations of Winzer, the filled diamond corresponds to the Hipparcos measurements, the filled circles are from the two seasons of observations by Adelman, and the filled square gives the result of the BV observations in this paper. The solid line denotes the line of constant period and the dotted line is the uncertainty.

Table 1. O-C derived from photometry and spectroscopy of HR 7224. $s$ denotes standard deviation in mmags.

\begin{tabular}{rrrrcl}
\hline \hline Year & \multicolumn{1}{c}{ O-C [d] } & $s$ & $N$ & Data & Source \\
\hline 1972 & $0.0580(70)$ & 4.4 & 48 & $U V B$ & Winzer (1974) \\
1991 & $-0.0010(60)$ & 11 & 409 & $B_{\mathrm{T}} V_{\mathrm{T}} H p$ & ESA (1997) \\
1994 & $-0.0005(20)$ & 3.8 & 317 & $u v b y$ & Adelman (1997) \\
1995 & $0.0005(18)$ & 3.3 & 299 & $u v b y$ & Adelman (1997) \\
2006 & $0.0341(24)$ & & 564 & $E W$ & Lehmann et al. \\
2008 & $0.0000(17)$ & 4.8 & 940 & $B V$ & this paper \\
\hline
\end{tabular}

the maximum of the Si equivalent width, $\Delta M_{0 \mathrm{EW}}$, relative to the light-curve maxima:

$$
\begin{aligned}
& P=1.1232524(6), M_{0}=2451582.7164(12), \\
& \Delta M_{0 \text { win }}=0.058(10), \Delta M_{0 \mathrm{EW}}=0.0341(32) .
\end{aligned}
$$

Our period agrees with those determined by ESA (1998) and Lehmann et al. (2006) within their uncertainties; the period appears to be constant over the past 17 years (see Fig. 2 and Table 1). The nonzero shift $\Delta M_{0 \mathrm{EW}}$ is likely connected with the fact that uneven horizontal distribution of silicon is not a unique source of the light variability (see Sect. 6.1). Winzer's (1974) $U B V$ light curves have the same shape as the more recent ones but are shifted with respect to the new ephemeris by $0.058(10)$ $(6 \sigma)$. Accepting the reality of the shift, we can speculate about the possibility of a transient rotational braking that might have occurred before the Hipparcos mission. We discussed a similar event in the He-strong star HD 37776 (Mikulášek et al. 2008b). In the case of HR 7224 however, we have no observational evidence of such deceleration between the Winzer and Hipparchos epochs. If we assume a constant rate of deceleration $(\dot{P}=$ const. $)$, then we need $\dot{P} \simeq 3.4 \times 10^{-9}$ in order to explain the of O-C shift of Winzer's observations. Such a deceleration would manifest itself in the lengthening of the period $P$ by $1.8 \mathrm{~s}$ during this time interval of 17 years (1972-1989).

The parameters $a_{1}, a_{2}$, which describe the shape of the normalised function $F\left(\vartheta, a_{1}, a_{2}\right)$ (Eq. (2)) show some minor 


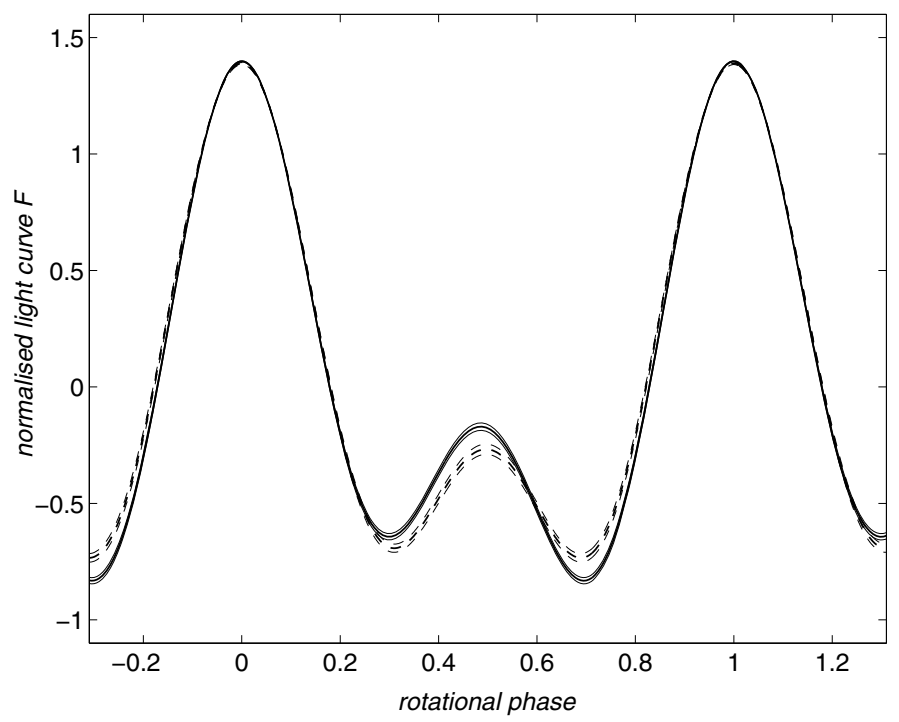

Fig. 3. Shapes of the normalised light curves of HR 7224 before 2000 (solid lines) and after 2000 (dashed lines). The outer light lines demarcate the uncertainty in the course of the light curves.

differences before and after the year 2000:

$$
\begin{array}{rll}
\text { before } 2000 & a_{1}=0.610(10) ; & a_{2}=0.084(12) ; \\
\text { after } 2000 & a_{1}=0.560(13) ; & a_{2}=0.018(15) .
\end{array}
$$

If real, this means that the secondary maximum of HR 7224 has been decreasing in brightness while the two minima have become nearly identical. It should be noted that the time dependence of parameters $a_{1}, a_{2}$ has no obvious effect on the star's period.

Changes in the shape of light curves are very rare among CP stars: the only star with well-documented light curve variations is the rapidly rotating silicon CP star 56 Arietis. The light curve variations are accompanied by a steady lengthening of the stellar period (Žižňovský et al. 2000; Adelman et al. 2001). On the other hand, in the most rapidly braked CP star known (HD 37776), we did not find any secular changes in its light curve (Mikulášek et al. 2008b). However, it is premature to speculate further on the nature of long-term light-curve variations of HR 7224 without further photometric and spectroscopic monitoring.

To compare the observed and calculated light-curve shapes and phases, we determined the shift between phases computed with our ephemeris and the ephemeris used by Lehmann et al. (2007) for surface mapping. The observed rotational variations of the silicon line equivalent widths demonstrated that Lehmann's phases precede ours by $0.1955(25)$. We have corrected for this phase difference in our analysis of the elemental surface distributions.

\section{Calculation of the light curve}

\subsection{Stellar parameters}

The stellar parameters of HR 7224 adopted in this study, as well as the abundances of silicon and iron derived from the abundance maps (see Fig. 4) and the abundance of helium are taken from Lehmann et al. (2006, 2007, see Table 2). The abundances are relative to hydrogen, $\varepsilon_{\mathrm{el}}=\log \left(N_{\mathrm{el}} / N_{\mathrm{H}}\right)$. Note that the abundances used by Lehmann et al. $(2006,2007)$ are defined slightly differently as $\log \left(N_{\mathrm{el}} / N_{\text {total }}\right)$.
Table 2. Stellar parameters of HR 7224.

\begin{tabular}{lc}
\hline \hline Effective temperature $T_{\text {eff }}$ & $14500 \mathrm{~K}$ \\
Surface gravity $\log g(\mathrm{cgs})$ & 4.2 \\
Inclination $i$ & $65^{\circ}$ \\
Helium abundance & $\varepsilon_{\mathrm{He}}=-3.5$ \\
Silicon abundance & $-3.6 \lesssim \varepsilon_{\mathrm{Si}} \lesssim-2.4$ \\
Iron abundance & $-4.4 \lesssim \varepsilon_{\mathrm{Fe}} \lesssim-3.0$ \\
\hline
\end{tabular}

\subsection{Model atmospheres and synthetic spectra}

We used the code TLUSTY (Hubeny 1988; Hubeny \& Lanz 1992, 1995; Lanz \& Hubeny 2003) for the model atmosphere calculations. We computed plane-parallel model atmospheres in LTE, taking into account the atomic data suitable for B type stars (Lanz \& Hubeny 2007). The atomic data for silicon are taken from Mendoza et al. (1995), Butler et al. (1993), and Taylor (2008), in preparation; for iron from Kurucz (1994), Nahar (1996), Nahar (1997), Bautista \& Pradhan (1997), and Bautista (1996), and for other elements from Luo \& Pradhan (1989), Fernley et al. (1999), Tully et al. (1990), Peach et al. (1988), Hibbert \& Scott (1994), and Nahar \& Pradhan (1993).

We assumed fixed values of the effective temperature and surface gravity and adopted models with a generic value $v_{\text {turb }}=$ $2 \mathrm{~km} \mathrm{~s}^{-1}$. We assumed the number density of helium relative to hydrogen to be $\log \left(N_{\mathrm{He}} / N_{\mathrm{H}}\right)=-3.5$ (Lehmann et al. 2007). The abundance of silicon and iron differed in individual models as explained below. We used the solar abundance of other elements according to Asplund et al. (2005).

For the spectrum synthesis (from which we calculate the photometric colours), we used the SYNSPEC code. We took into account the same transitions as for the model atmosphere calculations, and we also included the lines of all elements with the atomic number $Z \leq 30$ not included in the model atmosphere calculation. We computed angle-dependent intensities for 20 equidistantly spaced values of $\mu=\cos \theta$, where $\theta$ is the angle between the normal to the surface and the line of sight.

The model atmospheres and angle-dependent intensities $I\left(\lambda, \theta, \varepsilon_{\mathrm{Si}}, \varepsilon_{\mathrm{Fe}}\right)$ were calculated for a two-parametric grid of silicon and iron abundances $\varepsilon_{\mathrm{Si}}=\log \left(N_{\mathrm{Si}} / N_{\mathrm{H}}\right)=-4.0,-3.5,-3.0$, -2.5 , and -2.0 , and $\varepsilon_{\mathrm{Fe}}=\log \left(N_{\mathrm{Fe}} / N_{\mathrm{H}}\right)=-4.5,-4.0,-3.5$, and -3.0 .

\subsection{Phase dependent magnitude}

The radiative flux observed at the distance $D$ from the star with radius $R_{*}$ in a colour $c$ is calculated as

$f_{c}=\left(\frac{R_{*}}{D}\right)^{2} \int_{\substack{\text { visible } \\ \text { surface }}} I_{c}(\theta, \Omega) \cos \theta \mathrm{d} \Omega$

where the intensity $I_{c}(\theta, \Omega)$ at each surface point with surface spherical coordinates $\Omega$ is obtained by means of interpolation between the intensities ${ }^{1} I_{c}\left(\theta, \varepsilon_{\mathrm{Si}}, \varepsilon_{\mathrm{Fe}}\right)$ calculated from the grid of synthetic spectra as

$I_{c}\left(\theta, \varepsilon_{\mathrm{Si}}, \varepsilon_{\mathrm{Fe}}\right)=\int_{0}^{\infty} \Phi_{c}(\lambda) I\left(\lambda, \theta, \varepsilon_{\mathrm{Si}}, \varepsilon_{\mathrm{Fe}}\right) \mathrm{d} \lambda$.

\footnotetext{
1 Note that the SYNSPEC code outputs the specific intensities per unit frequency (not per unit wavelength).
} 

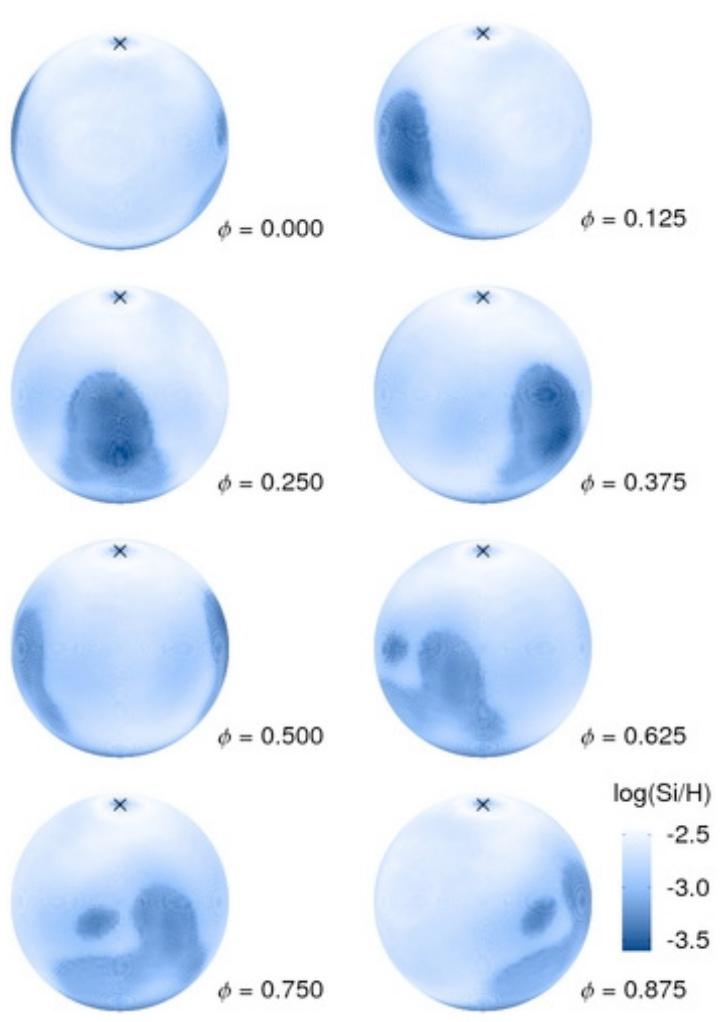
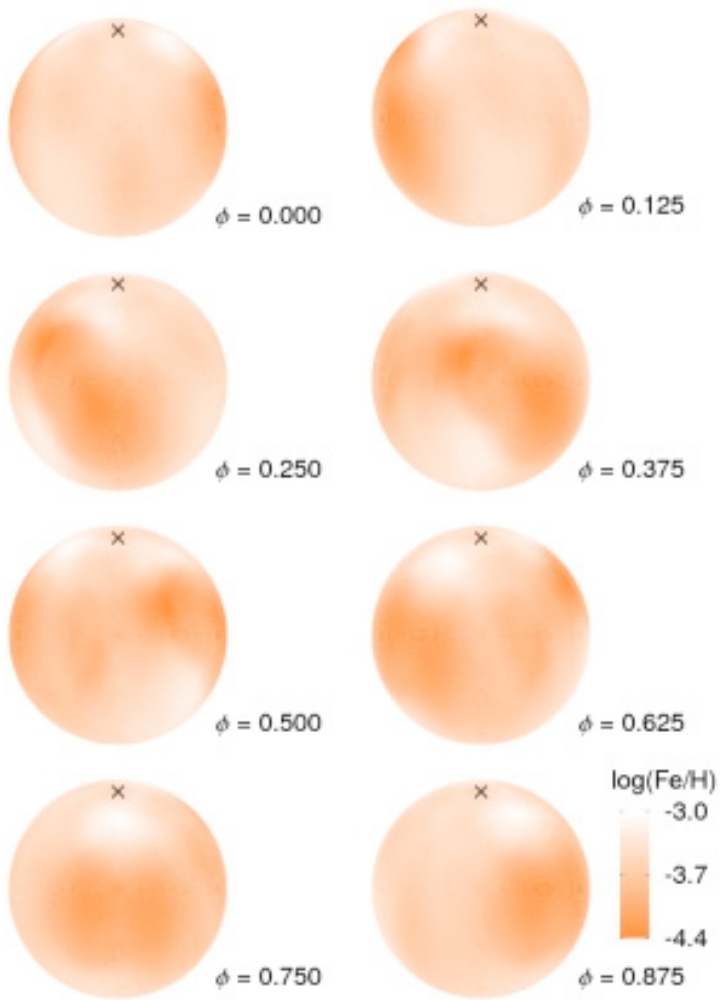

Fig. 4. Observed distribution of silicon (left) and iron (right) on the visible surface of HR 7224 at different rotational phases (Lehmann et al. 2007). Phases were calculated with the parameters given in Eq. (3).

Table 3. Central wavelengths and dispersions of the Gauss filter simulating the transmissivity functions.

\begin{tabular}{ccccccc}
\hline \hline Colour & $u$ & $v$ & $b$ & $g_{1}$ & $g_{2}$ & $y$ \\
\hline$\lambda_{a}[\AA]$ & 3500 & 4100 & 4700 & 5000 & 5220 & 5500 \\
$\sigma_{a}[\AA]$ & 230 & 120 & 120 & 65 & 65 & 120 \\
\hline
\end{tabular}

The transmissivity function $\Phi_{c}(\lambda)$ of a given filter $c$ of the Strömgren and $\Delta a$ photometric systems is approximated for simplicity by a Gauss function that peaks at the central wavelength of the colour $\lambda_{c}$ with dispersion $\sigma_{c}$ (see Table 3 ). The values for the uvby (Strömgren) photometric system are taken from Cox (2000). Values for the $\Delta a$ system (colours $g_{1}$ and $g_{2}$ ) are taken from Kupka et al. (2003).

The observed magnitude difference is

$\Delta m_{c}=-2.5 \log \left(\frac{f_{c}}{f_{c}^{\text {ref }}}\right)$

where $f_{c}$ is calculated from Eq. (5) and $f_{c}^{\text {ref }}$ is the reference flux obtained under the condition that the mean magnitude over all phases is zero.

\section{Influence of abundance anomalies on emergent fluxes}

As shown in Fig. 5, the enhanced silicon abundance results in the increase of the temperature in the continuum-forming region (within $\tau_{\text {ross }} \approx 0.1-1$ ) of the model atmosphere. The increased temperature is caused by enhanced silicon opacity in the model atmosphere. To understand the contribution of bound-free and bound-bound (line) processes to the temperature increase, we calculated model atmospheres with enhanced silicon abundance but neglecting silicon line transitions ${ }^{2}$ (see also Fig. 5). Neglecting silicon line transitions does not significantly influence the atmospheric temperature; consequently, enhanced opacity due to silicon bound-free transitions is the main cause of the temperature increase.

The silicon bound-free transitions are important mainly in the ultraviolet (UV) spectral regions at wavelengths shorter than $1600 \AA$. In atmospheres with overabundant silicon, the shortwavelength part of the spectrum is redistributed to the longer wavelengths of the UV spectrum, and also to the visible spectral regions (see Fig. 6). Consequently, the silicon-rich spots are bright in the $u v b y$ colours and are dark in the ultraviolet bands with $\lambda \lesssim 1600 \AA$.

A similar situation occurs for iron overabundance. Enhanced iron abundance leads to an increased temperature in the continuum-forming region between $\tau_{\text {ross }} \approx 1$ and $\tau_{\text {ross }} \approx 5 \times 10^{-3}$ (see Fig. 5). Unlike silicon, enhanced iron abundance warms the atmosphere above $\tau_{\text {ross }} \gtrsim 5 \times 10^{-3}$ mainly by line transitions. On the other hand, the outermost regions $\left(\tau_{\text {ross }} \lesssim 10^{-4}\right)$ of the model with $\varepsilon_{\mathrm{Fe}}=-3$ are slightly cooler than those with lower iron abundance. This effect is also due to iron lines, as the temperature is higher in the model with neglected line opacity (see also Fig. 5). A similar effect was reported by Khan \& Shulyak (2007)

The influence of iron overabundance on the spectrum is more complicated than the influence of silicon overabundance, as shown in Fig. 7. There are several depressions in the UV spectral region caused by numerous iron lines, but the flux with $\lambda \gtrsim 2500 \AA$ increases with increasing iron abundance. Generally,

\footnotetext{
2 Note that the resonance structure of silicon bound-free transitions was included in this test.
} 

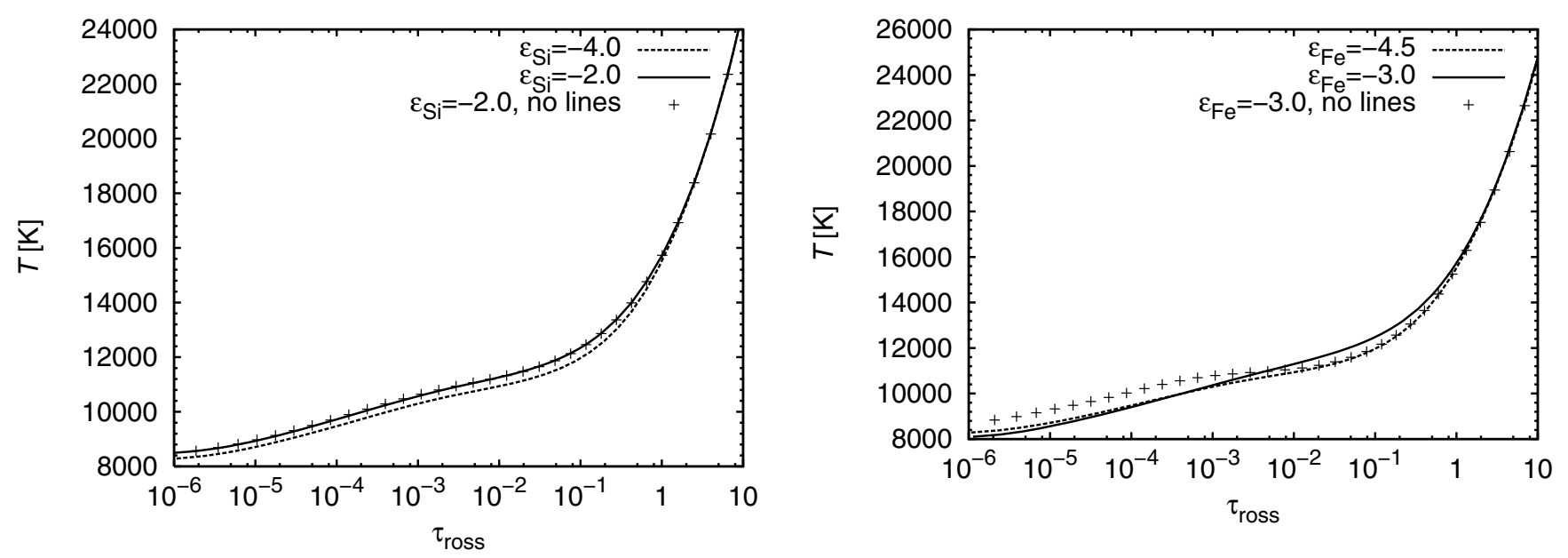

Fig. 5. The dependence of temperature on the Rosseland optical depth $\tau_{\text {ross }}$ in atmospheres with various chemical compositions. Left: influence of silicon abundance on the temperature for $\varepsilon_{\mathrm{Fe}}=-4.5$. Right: influence of iron abundance on the temperature for $\varepsilon_{\mathrm{Si}}=-4.0$. Crosses denote atmospheres with enhanced silicon or iron abundance, but neglecting the opacity due to line transitions of these elements.

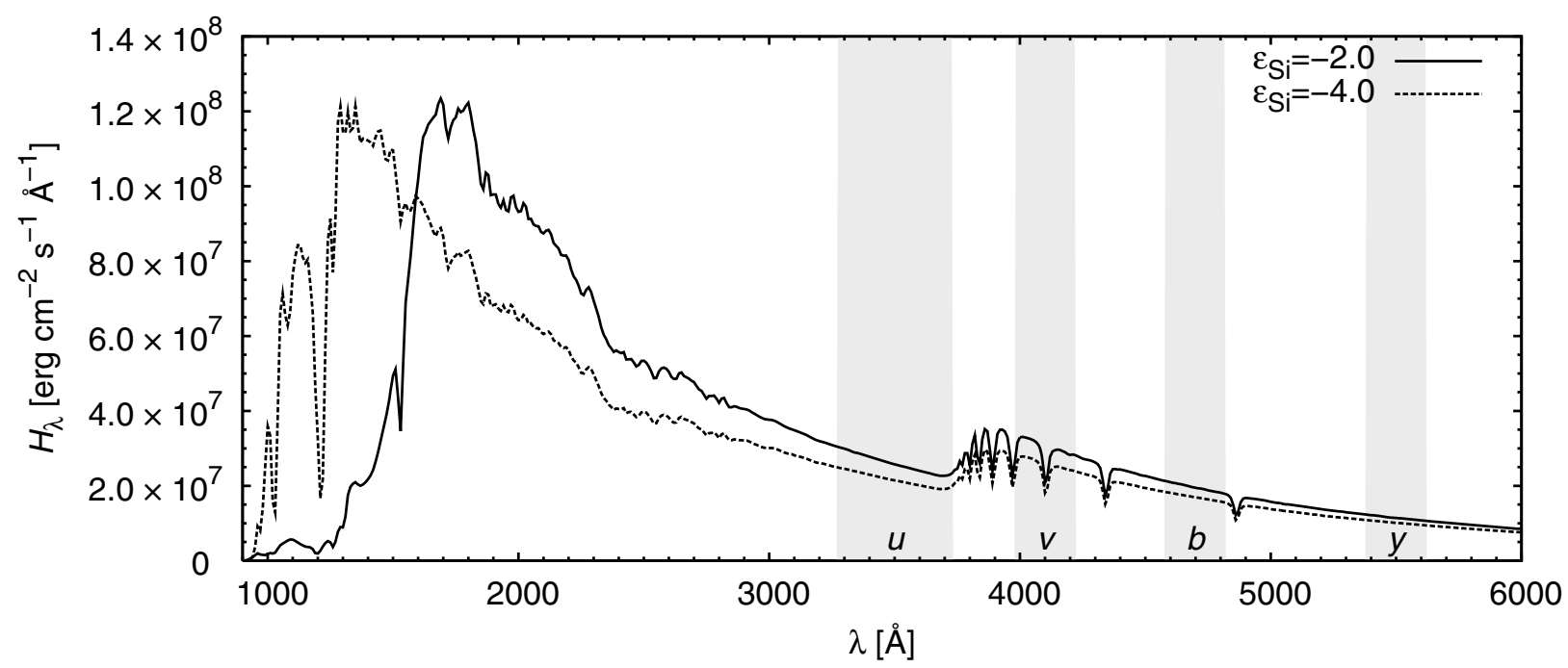

Fig. 6. The emergent flux from atmospheres with different silicon abundances. The flux was smoothed by a Gaussian filter with a dispersion of $10 \AA$ to show the changes in continuum, which are important for photometric variability. The passbands of the uvby photometric system are also shown on the graph (gray areas). The fluxes were calculated with TLUSTY for an iron abundance $\varepsilon_{\mathrm{Fe}}=-4.5$.

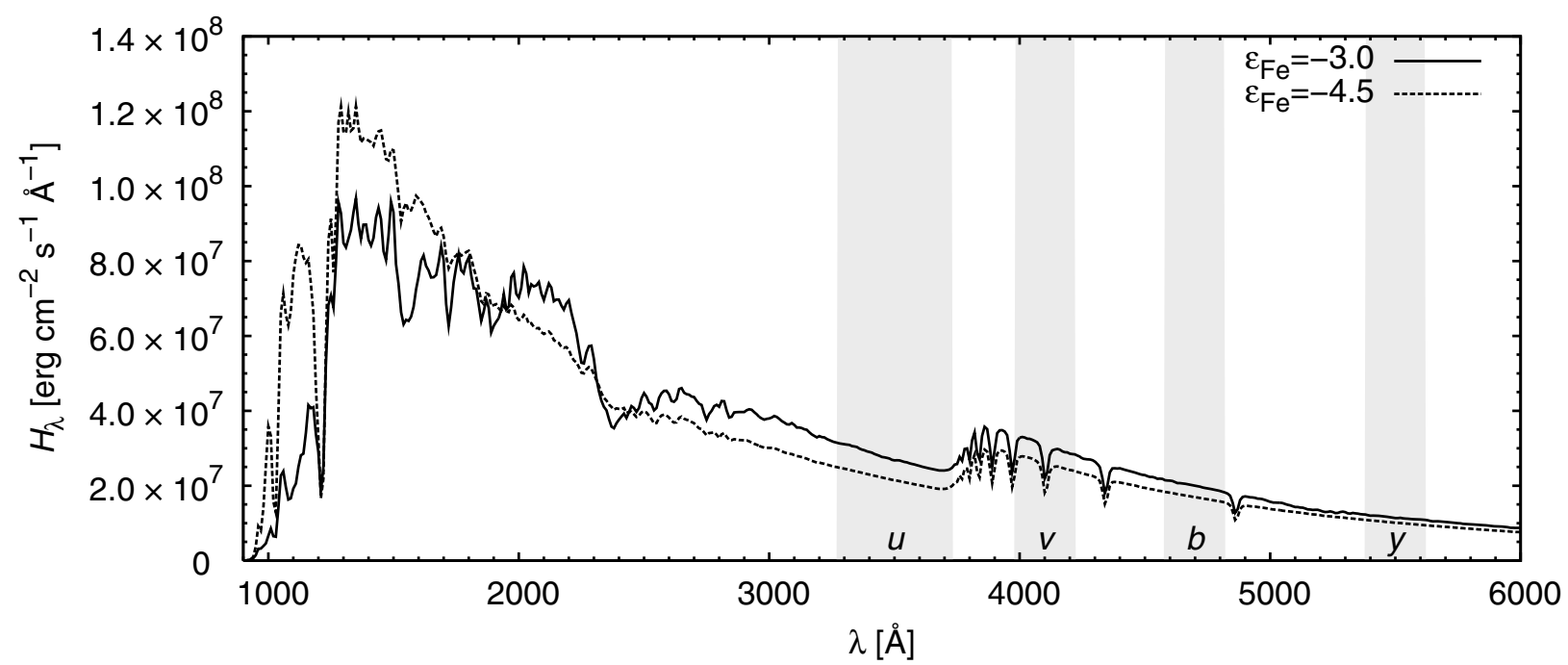

Fig. 7. The same as Fig. 6 but for iron. The fluxes were calculated with TLUSTY for a silicon abundance $\varepsilon_{S i}=-4.0$. 


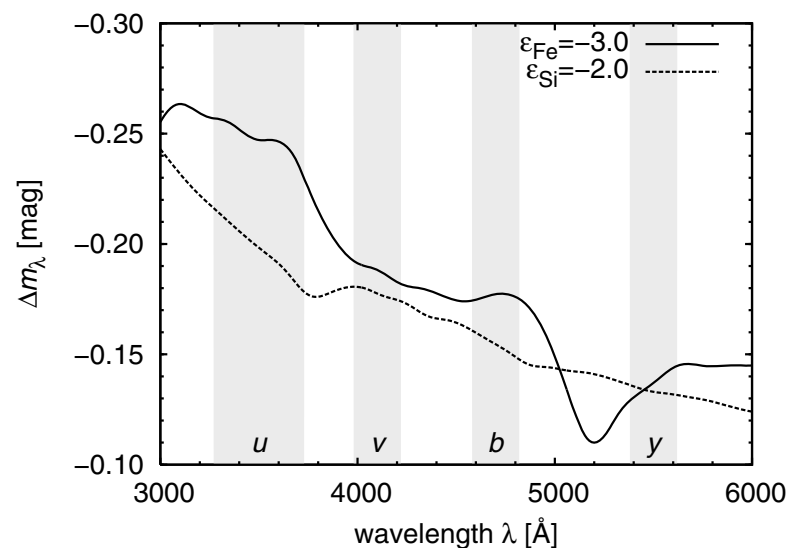

Fig. 8. The magnitude difference $\Delta m_{\lambda}$ (see Eq. (8)) between the emergent fluxes calculated with enhanced abundance of either silicon or iron and fluxes calculated assuming $\varepsilon_{\mathrm{Si}}=-4$ and $\varepsilon_{\mathrm{Fe}}=-4.5$. The fluxes were heavily smoothed by a Gaussian filter with a dispersion of $100 \AA$. The depression at $5200 \AA$ due to numerous iron lines is clearly apparent.

the iron line transitions redistribute the emergent UV radiation with $\lambda \lesssim 1700 \AA$ primarily to the long wavelength part of the spectrum with $\lambda \gtrsim 2500 \AA$. Consequently, iron-rich regions are bright in the uvby colours, whereas they are dark in the ultraviolet bands with $\lambda \lesssim 1700 \AA$.

These flux changes can be detected as a change in the apparent magnitude of the star (see Fig. 8). Here we plot the relative magnitude difference defined as

$\Delta m_{\lambda}=-2.5 \log \left(\frac{H_{\lambda}\left(\varepsilon_{\mathrm{Si}}, \varepsilon_{\mathrm{Fe}}\right)}{H_{\lambda}^{\mathrm{ref}}}\right)$,

where $H_{\lambda}^{\text {ref }}$ is the reference flux for $\varepsilon_{\mathrm{Si}}=-4.0, \varepsilon_{\mathrm{Fe}}=-4.5$. For both silicon and iron overabundance models, the absolute value of the relative magnitude difference decreases with increasing wavelength. For the silicon model, the decrease is nearly featureless, whereas the iron model exhibits several depressions in $\Delta m_{\lambda}$ due to the cumulation of iron lines. The most prominent feature at about $5200 \AA$ contributes significantly to the $5200 \AA$ depression frequently observed in the spectra of CP stars. Khan $\&$ Shulyak (2007) arrived at the same conclusion.

\section{Predicted light variations}

Predicted light curves are calculated from the surface abundance maps derived by Lehmann et al. (2007) and from the emergent fluxes computed with the SYNSPEC code, applying Eq. (7) for individual rotational phases. Since Lehmann et al. (2007) provide surface maps with different regularisation parameters $\Lambda$, we selected a map with $\Lambda=0.0001$, which is the most detailed one, for our initial calculations.

To study the influence of silicon and iron separately, we first calculated the light variations due to silicon only, assuming a constant iron abundance of $\varepsilon_{\mathrm{Fe}}=-4.5$. The observed light maximum occurs at the same phase at which the silicon lines have their maximum strength $(\phi=0)$. As silicon rich regions are bright in visible bands, our predicted light maximum should also occur at this phase. Indeed, there is a good agreement of both the times of maxima and minima of our predicted light curve and the observed light curve from Adelman (1997), though the amplitudes are different (Fig. 9).

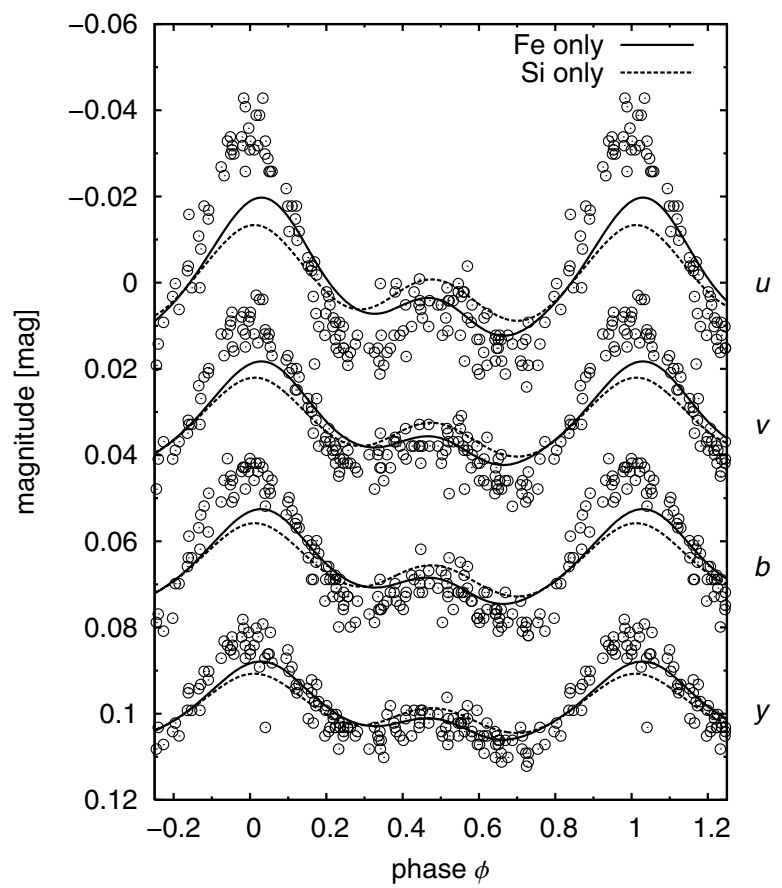

Fig. 9. Light variations of HR 7224 calculated from the silicon abundance maps only (dashed line) and from the iron abundance maps only (solid line). For both cases, $\Lambda=0.0001$. Observed light variations (open circles) are taken from Adelman (1997).

A similar test was performed using only the iron abundance map. The silicon abundance was assumed to be $\varepsilon_{\mathrm{Si}}=-4.0$. The iron lines are also observed to have their maximum strength close to phase $\phi=0$, i.e., during observed light maximum. As the iron-rich regions are bright in the uvby colours (see Fig. 7), the predicted light curve due to iron abundance variations alone also has a light maximum at phase $\phi=0$, in agreement with the observations. The predicted light curves due to iron only, displayed in Fig. 9, also have lower amplitudes than the observed ones.

Including the surface distribution of both silicon and iron in the calculation of the light curves (Fig. 10), we obtain very good agreement between the observed and predicted light curves in the $v, b$ and $y$ wavelength bands. In the $u$ band, however, the observed maximum is higher and the first minimum is deeper than predicted. A similar situation is seen in the colour indexes: $(v-b)$ and $(b-y)$ agree well with the observations while the $(u-b)$ curve is not a good fit, due to the differences in the $u$ band fit (Fig. 11). The observed Balmer discontinuity index $c_{1}$ exhibits large scatter due to the combination of the noisy $u, v$ and $b$ light curves, but its variation is nonetheless in accordance with the observations of Lehmann et al. (2007) on a possible variability of $\mathrm{H} \beta$ line. Similarly, the $m_{1}$ index, which reveals the effect of the metallic bound-bound absorption in the violet when compared with the $b$ and $y$ regions, shows a phase-dependence. The differences between the predicted curves of $c_{1}$ and $m_{1}$ and the observed ones clearly point to the existence of an additional, unknown mechanism working in the violet band that still has to be investigated (Fig. 10, and see also Sect. 6.1).

Thus, the inhomogeneous surface distribution of silicon and iron revealed in spectroscopic observations results in the appearance of spots on the stellar surface causing the photometric variability (see Fig. 12). 


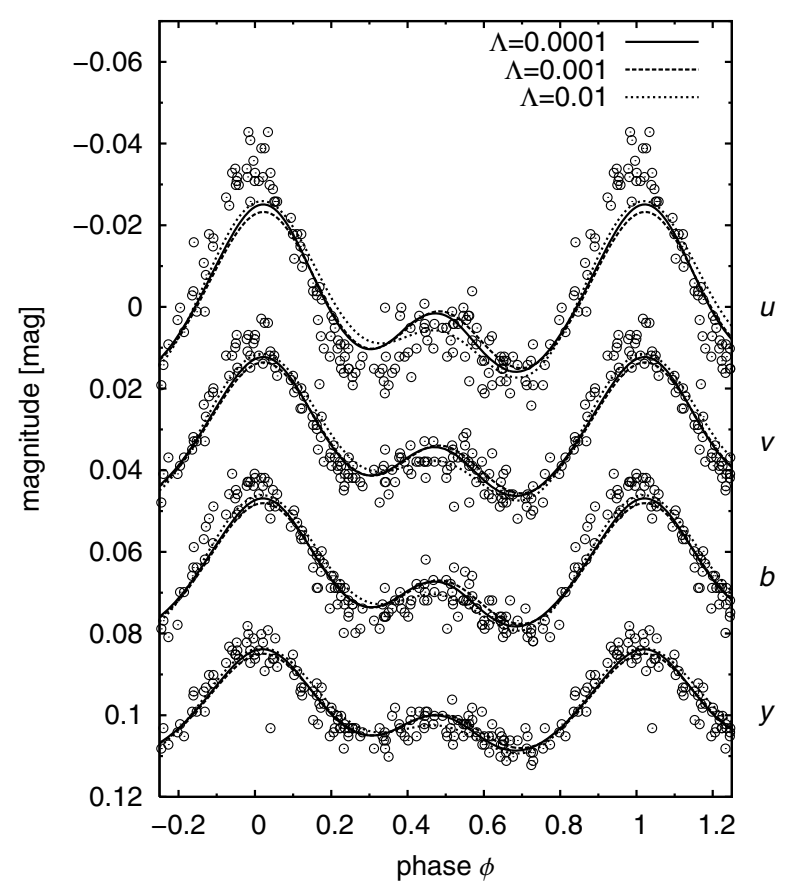

Fig. 10. Predicted light variations of HR 7224 computed taking into account both the silicon and iron surface abundance distributions derived by Lehmann et al. (2007) for different regularisation parameters $\Lambda$. Observed light variations (circles) are taken from Adelman (1997).

\section{Discussion}

\subsection{Detailed comparison of observed and predicted light curves}

The predicted and observed light curves differ slightly in their shapes (Fig. 10) and amplitudes (Fig. 13). The small differences in the observed vs. calculated amplitudes lead to the differences in the observed vs. calculated colour indexes, especially for $c_{1}$ and $m_{1}$ (see Fig. 11). The difference between the observed and predicted light curves in Fig. 10 could be explained by the presence of an additional photometric spot(s) on HR 7224 caused by overabundance of other element(s). We suspect chromium or magnesium. Khan \& Shulyak (2007) suggested chromium as an element capable of significant influence over the emergent flux from a star. On the other hand, Lehmann et al. (2006) found variability in the magnesium lines of HR 7224 with maximum strength occurring roughly at the same phase as the maximum difference between theory and observation in Fig. 10 (slightly before the maximum strength of silicon lines). This, along with the fact that the calculated effect of the magnesium abundance on the emergent flux has a significantly larger amplitude in the $u$ band rather than in $v, b$ and $y$, argues for magnesium as the cause of the residuals between observed and calculated light curves when considering only silicon and iron. Note however, that the maximum magnesium abundance derived by Lehmann et al. (2006) without surface mapping is too small to affect the spectral energy distribution significantly. The same is also true for helium and oxygen.

Lehmann et al. (2007) derived three abundance maps calculated with a different regularisation parameter, $\Lambda$, corresponding to a different assumed minimum size of the surface inhomogeneities. The shapes of the light curves calculated using the abundance maps obtained with a different value of $\Lambda$ are similar (see Fig. 10). A detailed comparison of these curves shows that

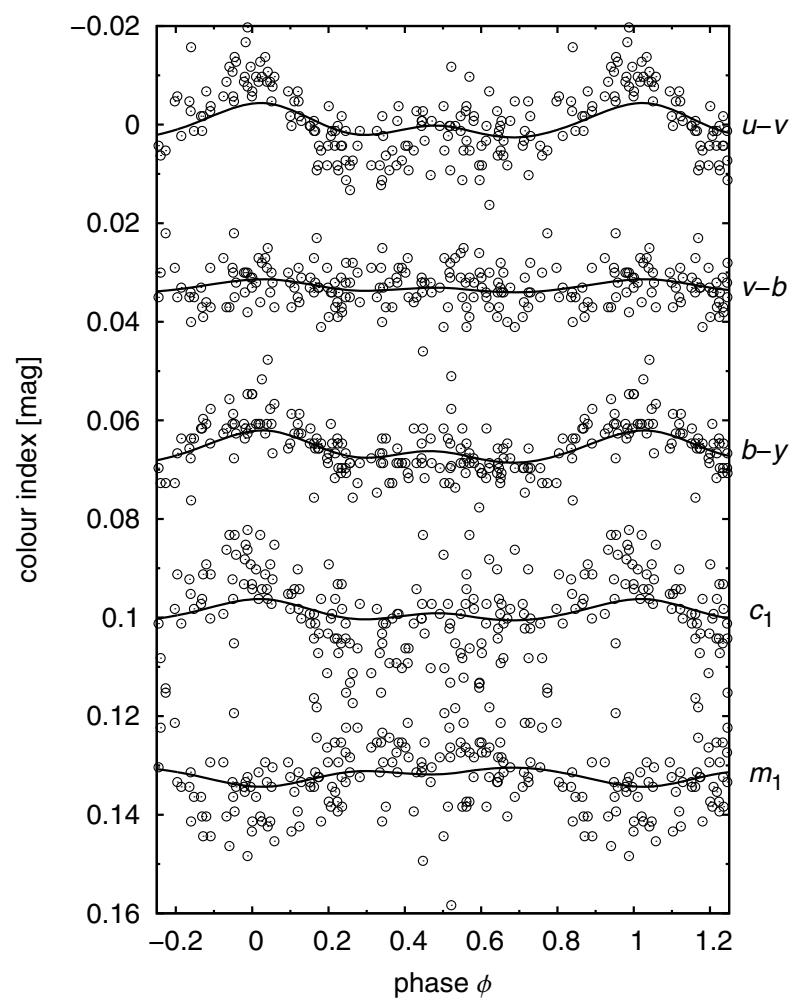

Fig. 11. Predicted variations of colour indexes (solid line) calculated from the surface abundance maps derived with $\Lambda=0.0001$ compared with the observations.

better agreement between theory and observation is obtained for the most complex surface map, i.e., for $\Lambda=0.0001$. However, the difference between observed and predicted light curves is significantly larger than the difference among the individual predicted curves calculated with different values of $\Lambda$.

\subsection{Model assumptions}

There are several effects that can influence the predicted light curves (see Krtička et al. 2007 for a more detailed discussion). For example, NLTE effects may influence the continuum flux distribution, but since HR 7224 surface maps were derived using LTE models, we confine ourselves to LTE models only. Strong surface magnetic field also influences the emergent spectral energy distribution (e.g., Khan \& Shulyak 2006). However, as all available measurements of surface magnetic field are negative and the corresponding upper limit is too low to signicantly influence the emergent flux, we neglect the influence of magnetic field.

HR 7224 is classified as a helium-weak chemically peculiar star, so a question could arise concerning the effect of the uneven surface distribution of helium on the light curve. In Krtička et al. (2007) we showed that helium influences the spectral energy distribution only in the case when it is overabundant (with respect to solar), so we neglect any possible inhomogeneous helium surface distribution in our model. We also tested how accumulation of $\mathrm{He}$ in the sub-photospheric layers affects emergent flux and draw a conclusion that for $\tau_{\text {ross }}>1$ this has no effect on the light variability. 


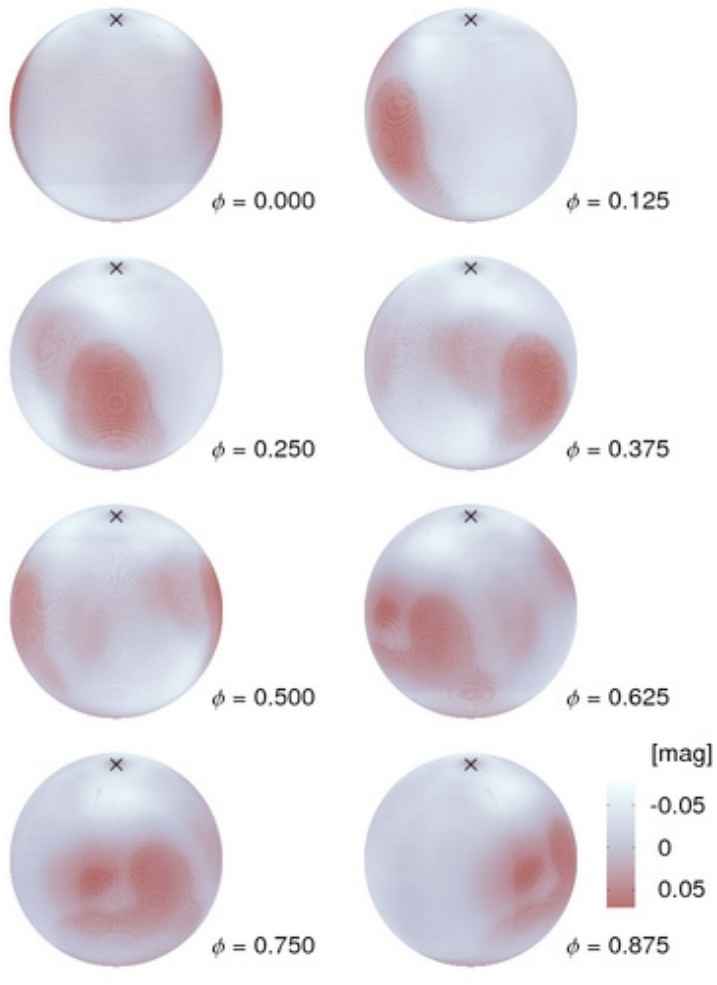

Fig. 12. The emergent intensity (in the $y$ band, $\mu=1$ ) from individual surface elements of HR 7224 at various rotational phases.

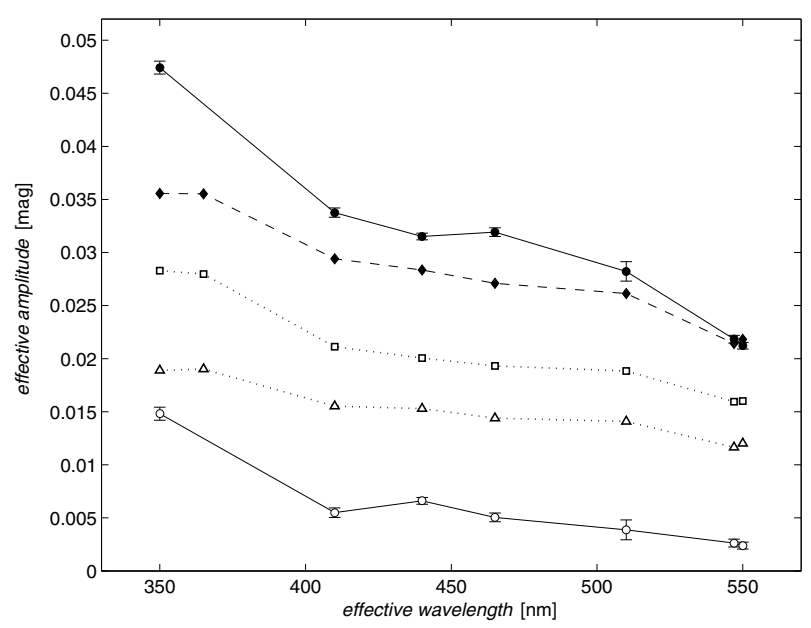

Fig. 13. The effective amplitudes of the predicted and observed light curves plotted versus the effective wavelengths of the various bands. The observed light-curve amplitudes are plotted with filled circles $(\bullet)$. The predicted amplitudes calculated using both silicon and iron maps are filled diamonds $(\downarrow)$, amplitudes calculated using iron only are open squares, $(\square)$, silicon only are triangles $(\triangle)$, and finally the residuals between observation and theory as open circles (o). Observed amplitudes in $B_{\mathrm{T}} V_{\mathrm{T}}$ and $U$ are not plotted here due to their large uncertainty.

\subsection{UV variations}

The redistribution of the flux from short to longer wavelengths is one of the consequences of the proposed mechanism for light variability in HR 7224. From our calculated model fluxes in Figs. 6 and 7, we predict that the star should have a flux minimum in the short-wavelength $\lambda \lesssim 1600 \AA$ part of the spectrum during the visible light maximum and vice versa. A similar antiphase behaviour of the short-wavelength UV and optical

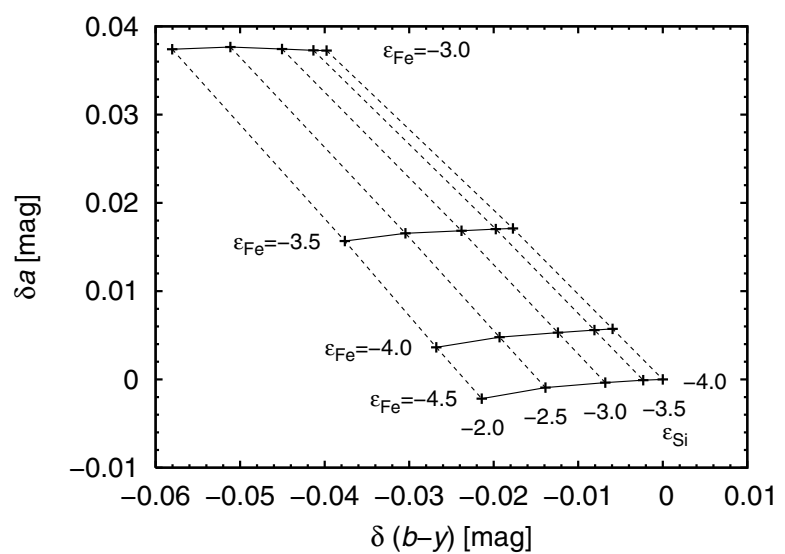

Fig. 14. The dependence of the $\delta a$ index on $\delta(b-y)$ for individual models from the model grid. The $\delta a$ values for models with the same iron abundance are connected with solid lines, and $\delta a$ values for the models with the same silicon composition are connected with dashed lines.

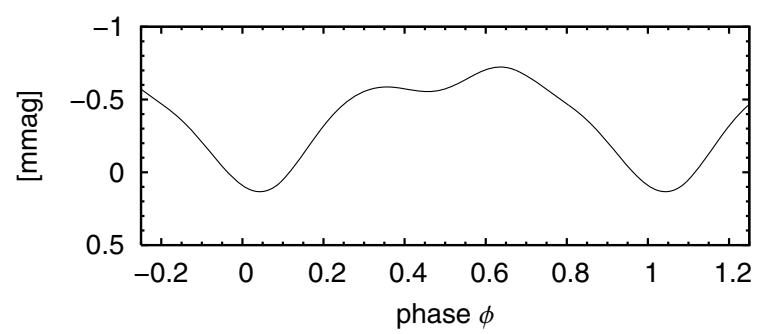

Fig. 15. The light curve in $\delta a$ index.

light curves has been reported for other CP stars (e.g., Sokolov 2006).

Unfortunately, there is no UV light curve of HR 7224 available. However, the star was observed in UV by the TD1 spacecraft (Jamar 1977, 1978). We note that our fluxes calculated with overabundant iron in Fig. 7 are in qualitative agreement with those observed by TD1 at different phases. In accordance with observations, the maximum UV light amplitude occurs at about $1400 \AA$, and the variations at this wavelength are anticorrelated with the variations in the visible. The variations at 1600-1900 ̊ are very small, and the variations at 2100-2300 are inversely correlated with that at $1400 \AA$. The absence of observed light variations at $2740 \AA$ may be connected with strong iron lines in this spectral region. Finally, the observed amplitude of the light variations at $1400 \AA$ (which is 0.24 mag, Jamar 1978) is in very good agreement with those derived from our models (0.21 mag for a Gaussian filter with dispersion $50 \AA$ ).

\subsection{Variations of a}

The peculiarity index $a$ introduced by Maitzen (1976) as

$a=g_{2}-\left(g_{1}+y\right) / 2$

is based on the existence of flux depressions (Kodaira 1969) correlated with the chemical peculiarity of a given star. To test whether the peculiarity demonstrated in the $a$-index is indeed caused by numerous iron lines (Khan \& Shulyak 2007), we calculated the peculiarity index $a$ for individual model atmospheres used in our previous calculations.

The dependence of $a$ on the iron and silicon abundance can be seen in Fig. 14, where we plot the dependence of $\delta a$ on 


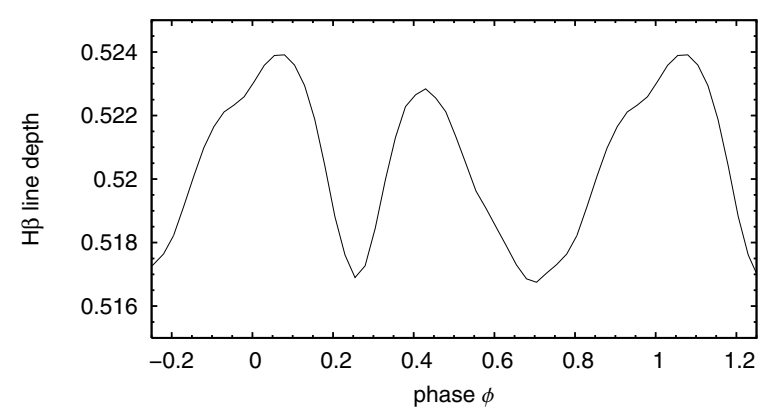

Fig. 16. The calculated variations of $\mathrm{H} \beta$ line depth with phase.

$\delta(b-y)$. These indices are defined relative to their values for the model with $\varepsilon_{\mathrm{Si}}=-4.0$ and $\varepsilon_{\mathrm{Fe}}=-4.5$,

$$
\begin{aligned}
\delta a & =a-a_{\varepsilon_{\mathrm{Si}}=-4.0, \varepsilon_{\mathrm{Fe}}=-4.5}, \\
\delta(b-y) & =(b-y)-(b-y)_{\varepsilon_{\mathrm{Si}}=-4.0, \varepsilon_{\mathrm{Fe}}=-4.5} .
\end{aligned}
$$

With increasing iron abundance, the $\delta a$ index increases, whereas the influence of silicon on $\delta a$ is only marginal. On the other hand, both the silicon and iron abundances influence the value of the $\delta(b-y)$ index. This implies that for a star with a given effective temperature (and an assumed homogeneous surface distribution of elements), it is possible to infer the value of iron and silicon abundance directly from photometry. One has to keep in mind, however, that other elements may also influence the value of $\delta(b-y)$ and $\delta a$ (cf., Kupka et al. 2004; Khan \& Shulyak 2007). Consequently, such procedure is likely possible only for hot CP stars. Finally, for stars with a strong magnetic field, the Zeeman splitting of iron lines may also influence the $a$ index (Khan \& Shulyak 2006).

As the star rotates, surface regions with different chemical composition appear on the visible disc and consequently $\delta a$ and $\delta(b-y)$ also vary with rotational phase (see Figs. 11 and 15). Here we plot the light curve calculated as described in Sect. 3.3 with filter parameters taken from Table 3. From this plot, we can conclude that the amplitudes of $\delta a$ variations are very small, on the order of millimagnitudes.

\subsection{H $\beta$ line profile variations}

The fact that we are able to predict the correct amplitudes of brightness variations in both the UV and visual spectral domains from just the abundance maps of iron and silicon supports the conclusion that the effective temperature is constant on the surface of HR 7224. This finding weakens one of possible explanations of Balmer lines' profile variations as summarised by Lehmann et al. (2007). Since the effect of the Lorenz force on the line profiles is mainly on the line wings (cf., Shulyak et al. 2007), it cannot explain the line profile variability observed in HR 7224. We tested if our model is also able to explain the observed $\mathrm{H} \beta$ line variability. For this purpose we calculated the line profiles in individual phases using the code developed by Skalický (2008). The code numerically integrates the emergent intensity $I_{c}(\theta, \Omega)$ over the visible stellar surface $\Omega$ taking into account the Doppler shift due to the stellar rotation. The emergent intensity from each surface point is calculated by interpolating the synthetic spectra $I\left(\lambda, \theta, \varepsilon_{\mathrm{Si}}, \varepsilon_{\mathrm{Fe}}\right)$ for appropriate surface abundance distribution of silicon and iron (Lehmann et al. 2007). We assume fixed effective temperature and surface gravity. The predicted phases of the maximum line depth (Fig. 16) roughly correspond to the observed ones (Lehmann et al. 2006, Fig. 13 therein). Also the shape of the minimum at the phase $\phi \approx 0.2$ is roughly correct. However, the observed amplitude of the minimum at the phase $\phi \approx 0.6$ seems to be a factor of about 2 higher. We conclude that either another element has to play a role in the $\mathrm{H} \beta$ line variability, or the observed difference between observation and theory is partially caused by noisy observational data.

\section{Conclusions}

We successfully simulated the light variability of HR 7224 directly from the silicon and iron surface abundance maps derived by Lehmann et al. (2007). There is very good agreement between the observed and predicted light variability in the uvby bands of the Strömgren photometric system. We did not introduce any free parameter to improve the agreement between the theoretical and observed light curves.

The rotationally modulated light variability of HR 7224 is caused by the flux redistribution due to iron line transitions, silicon bound-free transitions, and by the inhomogeneous surface distribution of these elements. This picture is also supported by the agreement between the predicted behaviour of the UV flux distribution and that observed by the TD1 satellite.

We support the conclusion of Khan \& Shulyak (2007) that numerous iron lines contribute significantly to the well-known depression at $5200 \AA$. Moreover, we show that iron is able to influence the peculiarity index $a$, whereas silicon's influence is marginal. With a suitable calibration, this could enable the derivation of abundances in hot $\mathrm{CP}$ stars directly from photometry.

We conclude that a promising explanation for the light variations in CP stars is a flux redistribution through line and boundfree transitions combined with the inhomogeneous surface distribution of various elements.

Acknowledgements. We thank Dr. A. Tkachenko for providing us with surface maps and Drs. E. Paunzen and D. Shulyak for the discussion of this topic. This work was supported by grants GA ČR 205/06/0217, GA ČR 205/08/H005, VEGA 2/6036/6, MEB 080832/SK-CZ-0090-07, MEB 060807, and partly by GAČR 205/07/0031. This research made use of NASA's Astrophysics Data System, the SIMBAD database, operated at the CDS, Strasbourg, France and the on-line database of photometric observations of mCP stars (Mikulášek et al. 2007a). G.W.H. acknowledges support from NASA, NSF, Tennessee State University, and the State of Tennessee through its Centers of Excellence program.

\section{References}

Adelman, S. J. 1997, A\&A, 122, 249

Adelman, S. J. 2004, MNRAS, 351, 823

Adelman, S. J., Malanushenko, V., Ryabchikova, T. A., \& Savanov, I. 2001, A\&A, 375, 982

Asplund, M., Grevesse, N., \& Sauval, A. J. 2005, Cosmic Abundances as Records of Stellar Evolution and Nucleosynthesis, ed. T. G. Barnes III, \& F. N. Bash (San Francisco: ASP), ASP Conf. Ser., 336, 25

Bautista, M. A. 1996, A\&AS, 119, 105

Bautista, M. A., \& Pradhan, A. K. 1997, A\&AS, 126, 365

Bohlender, D. A., Landstreet, J. D., \& Thompson, I. B. 1993, A\&A, 413, 273

Butler, K., Mendoza, C., \& Zeippen, C. J. 1993, J. Phys. B, 26, 4409

Cowley, A., Cowley, C., Jaschek, M., et al. 1969 AJ, 74, 375

Cox, A. N. 2000, Astrophysical Quantities (New York: AIP Press)

Eaton, J. A., Henry, G. W., \& Fekel, F. C. 2003, in The Future of Small Telescopes in the New Millennium, Vol. II, The Telescopes We Use, ed. T. D. Oswalt (Dordrecht: Kluwer), 189

ESA 1997, The Hipparcos and Tycho Catalogs, SP-1200

ESA 1998, The Hipparcos and Tycho Catalogs, Celestia 2000, SP-1220

Fernley, J. A., Hibbert, A., Kingston, A. E., \& Seaton, M. J. 1999, J. Phys. B, 32, 5507

Henry, G.W. 1995a, in Robotic Telescopes: Current Capabilities, Present Developments, and Future Prospects for Automated Astronomy, ed. G. W. Henry, \& J. A. Eaton (San Francisco: ASP), ASP Conf. Ser., 79, 37 
Henry, G. W. 1995b, in Robotic Telescopes: Current Capabilities, Present Developments, and Future Prospects for Automated Astronomy, ed. G. W. Henry, \& J. A. Eaton (San Francisco: ASP), ASP Conf. Ser., 79, 44

Hibbert, A., \& Scott, M. P. 1994, J. Phys. B, 27, 1315

Hubeny, I. 1988, Comput. Phys. Commun., 52, 103

Hubeny, I., \& Lanz, T. 1992, A\&A, 262, 501

Hubeny, I., \& Lanz, T. 1995, ApJ, 439, 875

Jamar, C. 1977, A\&A, 56, 413

Jamar, C. 1978, A\&A, 70, 379

Khan, S. A., \& Shulyak, D. V. 2006, A\&A, 454, 933

Khan, S. A., \& Shulyak, D. V. 2007, A\&A, 469, 1083

Khokhlova, V. L., Vasilchenko, D. V., Stepanov, V. V., \& Romanyuk, I. I. 2000, AstL, 26, 177

Kochukhov, O., Khan, S., \& Shulyak, D. 2005, A\&A, 433, 671

Kodaira, K. 1967, Ann. Tokyo Astr. Obs., 10, 157

Kodaira, K. 1969, ApJ, 157, L59

Krivosheina, A. A., Ryabchikova, T. A., \& Khokhlova, V.L. 1980, Nauchnye Informatsii, Ser. Astrof., 43, 70

Krtička, J., Mikulášek, Z., Zverko, J., \& Zižňovský, J. 2007, A\&A, 470, 1089

Kupka, F., Paunzen, E., \& Maitzen, H. M. 2003, MNRAS, 341, 849

Kupka, F., Paunzen, E., Iliev, I. Kh., \& Maitzen, H. M. 2004, MNRAS, 352, 863

Kurucz, R. L. 1994, Kurucz CD-ROM 22, Atomic Data for Fe and $\mathrm{Ni}$ (Cambridge: SAO)

Landstreet, J. D., \& Borra, E. F. 1978, ApJL, 224, 5

Lanz, T., \& Hubeny, I. 2003, ApJS, 146, 417

Lanz, T., \& Hubeny, I. 2007, ApJS, 169, 83

Lanz, T., Artru, M.-C., Le Dourneuf, M., \& Hubeny, I. 1996, A\&A, 309, 218

Lehmann, H., Tsymbal, V., Mkrtichian, D. E., \& Fraga, L. 2006, A\&A, 457, 1033

Lehmann, H., Tkachenko, A., Fraga, L., Tsymbal, V., \& Mkrtichian, D. E. 2007, A\&A, 471, 941

Luo, D., \& Pradhan, A. K. 1989, J. Phys. B, 22, 3377
Maitzen, H. M. 1976, A\&A, 51, 223

Mendoza, C., Eissner, W., Le Dourneuf, M., \& Zeippen, C. J. 1995, J. Phys. B, 28,3485

Michaud, G. 2005, in The A-Star Puzzle, ed. J. Zverko, J. Žižňovský, S. J. Adelman, \& W. W. Weiss (Cambridge: Cambridge Univ. Press), IAU Symp., 224,173

Mikulášek, Z., Janík, J., Zverko, J., et al. 2007a, Astron. Nachr., 328, 10

Mikulášek, Z., Krtička, J., Zverko, J., et al. 2007b, in Physics of Magnetic Stars,

ed. I. I. Romanyuk, \& D. O. Kudryavtsev, SAO, Nizhnij Arkhyz, 300

Mikulášek, Z., Gráf, T., Krtička, J., et al. 2008a, CAOSP, 38, 363

Mikulášek, Z., Krtička, J., Henry, G. W., et al. 2008b, A\&A, 485, 585

Molnar, M. R. 1972, ApJ, 175, 453

Molnar, M. R. 1973, ApJ, 179, 527

Nahar, S. N. 1996, Phys. Rev. A, 53, 1545

Nahar, S. N. 1997, Phys. Rev. A, 55, 1980

Nahar, S. N., \& Pradhan, A. K. 1993, J. Phys. B, 26, 1109

Nakajima, R. 1985, Ap\&SS, 116, 285

Peach, G., Saraph, H. E., \& Seaton, M. J. 1988, J. Phys. B, 21, 3669

Peterson, D. M. 1970, ApJ, 161, 685

Seaton, M. J., Zeippen, C. J., Tully, J. A., et al. 1992, Rev. Mex. Astron. Astrofis., 23,19

Shaham, J. 1986, ApJ, 310, 780

Shulyak, D., Valyavin, G., Kochukhov, O., et al. 2007, A\&A, 464, 108

Skalický, J. 2008, Diploma Thesis, Masaryk University, Brno

Smith, M. A., \& Groote, D. 2001, A\&A, 372, 208

Sokolov, N. A. 2006, MNRAS, 373, 666

Townsend, R. H. D., Owocki, S. P., \& Groote D. 2005, ApJ, 630, L81

Tully, J. A., Seaton, M. J., \& Berrington, K. A. 1990, J. Phys. B, 23, 3811

Vauclair, S. 2003, Ap\&SS, 284, 205

Weiss, W. W., Albrecht, R., \& Wieder, R. 1976, A\&A, 47, 423

Winzer, J. E. 1974, Ph.D. Thesis, Univ. Toronto

Žižňovský, J., Schwartz, P., \& Zverko, J. 2000, IBVS, 4835 